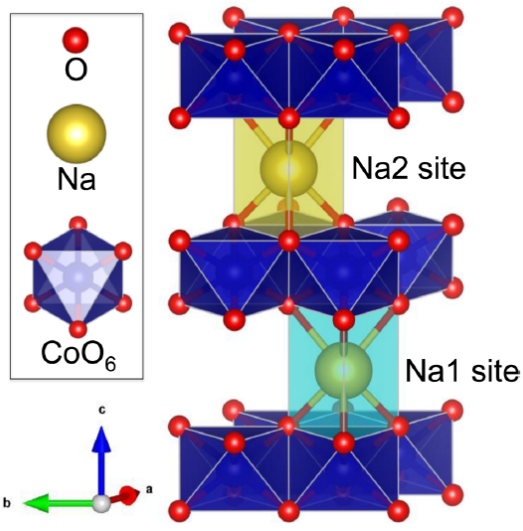

Figure 1. Crystal structure of $\mathrm{NaCoO}_{2}[1]$

Keywords: Na-ion battery

\section{MS19-P3 Molecular and Crystal Structures of Aminoalanes and Aminoboranes}

Thomas Bernert ${ }^{1}$, Morten B. Ley ${ }^{1}$, Alexander Bodach ${ }^{2}$, Javier Ruiz-Fuertes $^{3}$, Michael Fischer ${ }^{4,5}$, Michael Felderhoff ${ }^{1}$, Claudia Weidenthaler ${ }^{1}$

1. Max-Planck-Institut fuer Kohlenforschung, Kaiser-Wilhelm-Platz 1, D-45470 Muelheim an der Ruhr, Germany 2. Goethe-Universitaet Frankfurt am Main, Institut fuer Anorganische und Analytische Chemie, Max-von-Laue Straße 7, D-60438 Frankfurt am Main, Germany

3. Departament de Física Aplicada - ICMUV, Universitat de València, Dr. Moliner 50, E- 46100 Burjassot, Spain

4. Fachgebiet Kristallographie, Fachbereich Geowissenschaften, University of Bremen, Klagenfurter Straße, D-28359 Bremen, Germany

5. MAPEX Center for Materials and Processes, University of Bremen, Germany

email: bernert@mpi-muelheim.mpg.de

Compounds, bearing $\mathrm{H}^{\delta+}$ and $\mathrm{H}^{\delta-}$ species, are promising candidates for reversible hydrogen storage materials. This strategy has already been discussed, e.g. by Stephan and Erker [1] for Lewis-acid/base adducts, which show a reversible hydrogenation. Lewis-acid/base adducts of nitrogen and aluminium or boron could be economically and environmentally beneficial candidates for hydrogen storage, e.g. in $\mathrm{NH}_{3} \mathrm{BH}_{3}[2,3]$. Within the group 13 elements, Lewis-acid/base adducts with different substituents often form molecular structures build up by dimers or trimers [Figure 1 (a)]. Since, the reactivity of these compounds towards small molecules like $\mathrm{H}_{2}$ [4] or $\mathrm{CO}_{2}$ [5] strongly depend on the bond lengths between the corresponding Lewis acid and the base, a knowledge of their molecular structures is crucial for their applications.

Recently, we determined the crystal structure of diethylaminoalane by a combination of powder X-ray diffraction, Raman spectroscopy and DFT calculations [6]. This approach has led to a reliable molecular structure determination of diethylaminoalane in the solid state and to obtain further information about the environment and behavior of the Al-H bonds. This combination of techniques was also applied to characterise a phase transition of dimethylaminoalane [Figure 1 (b)] from a monoclinic to a plastic crystalline phase, where the molecules are found to be strongly disordered and arranged accordingly to the cubic a15 phase, often adopted by liquid crystals. The analogous boron compound, dimethylaminoborane, is an example for a substance which can exist as dimer or as a trimer. For dimeric dimethylaminoborane, we were able to determine its monoclinic crystal structure and the second order phase transition to a triclinic phase by single crystal $\mathrm{X}$-ray diffraction and in-situ X-ray powder diffraction. Our measurements confirm the cell parameters from [7], and led to the atomic positions.

\section{References:}

[1] D. W. Stephan, G. Erker (2010), Angew. Chem., Int. Ed., 49, 46 - 76 .

[2] A. Staubitz et al. (2010), Chem. Rev., 110, 4079 4124.

[3] P. P. Power (2010), Nature, 463, 171 - 177.

[4] Z. Xiong et al. (2008), Nat. Mater., 7, 138 - 141.

[5] J. A. B. Abdalla et al. (2015), Angew. Chem., Int. Ed., 54, 5098 - 5102 . 
[6] T. Bernert et al. (2016), Acta Crystallogr. B., 72, $232-240$

[7] P. J. Schapiro (1962), PhD Thesis, Refcode CSD: DMABDI.
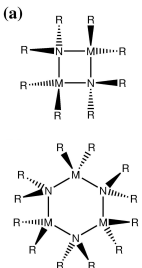

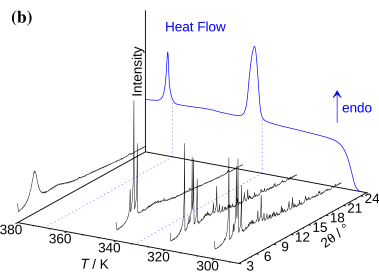

Figure 1. (a) Typical molecular structures of amino adducts of group 13 elements (M) with $\mathrm{R}=\mathrm{H}$ or alkyl moieties. (b) In-situ diffractograms during heating of dimethylaminoalane together with DSC data.

Keywords: Solid-State Hydrogen Storage, Phase Transitions, Molecular Crystals, Aminoalanes, Aminoboranes

\section{MS19-P4 Structural design principles for close packed $\mathrm{Na}$ and Li solid-electrolytes built from mixed anion borane lattices \\ Matteo Brighi ${ }^{1}$, Pascal Schouwink ${ }^{1}$, Yolanda Sadikin $^{1}$, Radovan
Cerny \\ 1. Laboratory of Crystallography, DQMP, University of Geneva, 24 Quai Ernest-Ansermet, CH-1211 Genève \\ email: matteo.brighi@unige.ch}

Energy storage solutions on a large scale have long been identified as a primary target within renewable energy research. While various complex hydrides, such as the salts built from the $\left[\mathrm{BH}_{4}\right]^{-}$anion, have shown high $\mathrm{Li}^{+}$ ionic conductivity, the compound family of the metal boranes are more promising contenders when it comes to Na-superionics and all-solid battery concepts. Very recently, the complex anion landscape of complex hydrides has been extended to include the carborane (CB) $\left[\mathrm{CB}_{11} \mathrm{H}_{12}\right]^{-}$. When used to template close-packed ionic conductors, they show the highest reported $\mathrm{Li}^{+}$and $\mathrm{Na}^{+}$ ionic conductivities of all reported complex hydrides ${ }^{1}$ and are amongst the highest for $\mathrm{Na}$ solid electrolytes in general. Due to their quasi-spherical shape, this molecule easily forms the cubic close packed structure ( $c c p)$, an hard backbone to tailor vacancy concentration achieving high ionic mobility ${ }^{2}$, which is boosted additionally by well-known "paddle wheel effect" 3 . We present different approaches to design in particular $\mathrm{Na}$-conducting solid electrolytes with ever lower operating temperatures. On the one hand, it is conceptually straightforward to rebuild benchmark conductors from the literature. In this sense, the ionic conductor $\operatorname{RbAg}_{4} \mathrm{I}_{5}$ was taken as a template to reproduce its $\mathrm{CB}$ analogues, resulting in double cation phases containing alkali metals, and with an ideal composition of $\mathrm{CsA}_{4}\left(\mathrm{CB}_{11} \mathrm{H}_{12}\right)_{5}\left(\mathrm{~A}=\mathrm{Li}^{+}, \mathrm{Na}^{+}\right)$in the specific case of the parent phase $\mathrm{RbAg}_{4} \mathrm{I}_{5}$ The ionic mobility of the resulting new phases will be presented and related to the crystal structure. On the other hand, we show how the close packing of the anion lattice may be controlled by anion-mixing of $\mathrm{CB}\left[\mathrm{CB}_{11} \mathrm{H}_{12}\right]^{-}$and dodeca-boranes $\left[\mathrm{B}_{12} \mathrm{H}_{12}\right]^{2-}$, both their Na-endmembers known to have superionic $h t$-phases. Making use of the knowledge of preferred coordination polyhedra in higher boranes, this approach allows us to control the occupancy of tetrahedral $(\mathrm{T})$ and octahedral $(\mathrm{O})$ vacancies by modifying the carborane - dodeca-borane ratio, made possible due to the different charges of the polyanions. A control of site occupancies in packed lattices is known to be the key point to achieve high ionic conductivities at a suitable temperature and therefore highly promising approach to tailor metal boranes for battery application.

References: 1. Tang et al Energy. Envrion. Sci., 2015, 8, 3637 2. Wang et al Nat. Mat. 2015, 14, 1026 3. Jansen Angew. Chem. Int. Ed. 1991, 30, 1547

Keywords: ionic conductor, solid electrolyte, Li battery, $\mathrm{Na}$ battery, closo-boranes, carboranes 